\title{
Practical Therapeutics
}

Drugs 40 (6): 854-862, 1990

0012-6667/90/0012-0854/\$04.50/0

(C) Adis International Limited

All rights reserved.

DRUG03 388

\section{The Sore Throat \\ When to Investigate and When to Prescribe}

\author{
S.D.R. Lang and K. Singh
}

Middlemore Hospital, Auckland

Contents

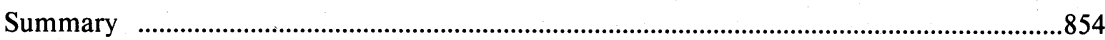

1. Aetiology, Clinical Diagnosis and Management of Sore Throat ……..............................855

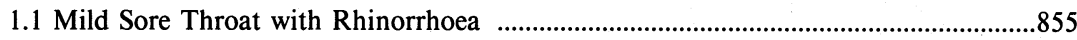

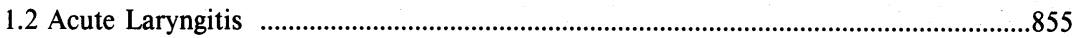

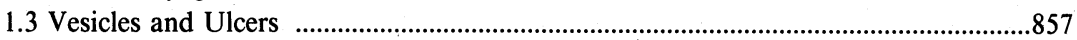

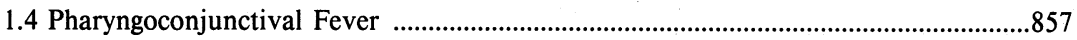

1.5 Pharyngitis with Influenza or Lower Respiratory Tract Infection ..............................857

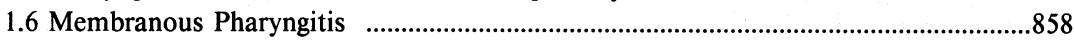

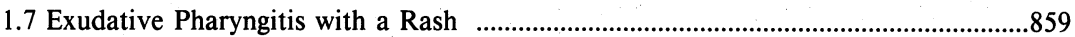

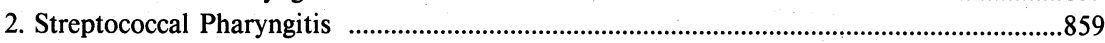

2.1 Investigation and Treatment Strategies ……………….............................................8.

2.1.1 Positive Throat Swab Culture ............................................................................860

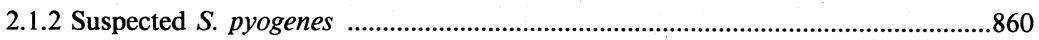

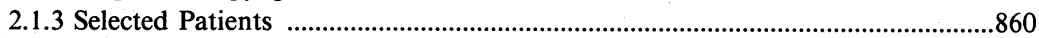

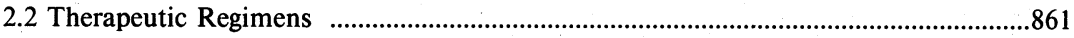

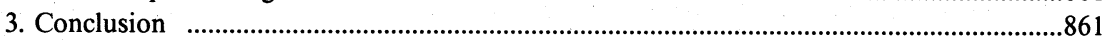

Sore throats are most commonly due to infections, many of which are viral and do not require specific treatment. Symptoms and signs of the common cold, influenza or croup, the occurrence of conjunctivitis in some adenoviral infections, generalised lymphadenopathy and splenomegaly in glandular fever or the presence of vesicles characteristic of herpangina (Coxsackie A virus) or of herpes simplex infection, occasionally enable a clinical diagnosis and avoid the need for antibiotic therapy. In the case of treatable conditions a typical membrane may suggest diphtheria, a scarlatiniform rash infection due to Streptococcus pyogenes or to Corynebacterium haemolyticum, and a cherry-red epiglottis Haemophilus influenzae type b. Associated atypical pneumonia suggests infection with Mycoplasma pneumoniae or Chlamydia pneumoniae. Pharyngitis due to Neisseria gonorrhoeae may be accompanied by infection at other sites or by other sexually transmitted diseases. Candidal infection, in the appropriate clinical circumstance, should suggest HIV infection. Surgical drainage is required in the case of peritonsillar or retropharyngeal abscess. Noninfectious cases of sore throat, e.g. thyroiditis, are relatively uncommon considerations in the differential diagnosis of acute febrile pharyngitis. The most common problem is to recognise streptococcal pharyngitis, which requires antibiotic treatment for 10 days to avoid the risk of rheumatic fever. 


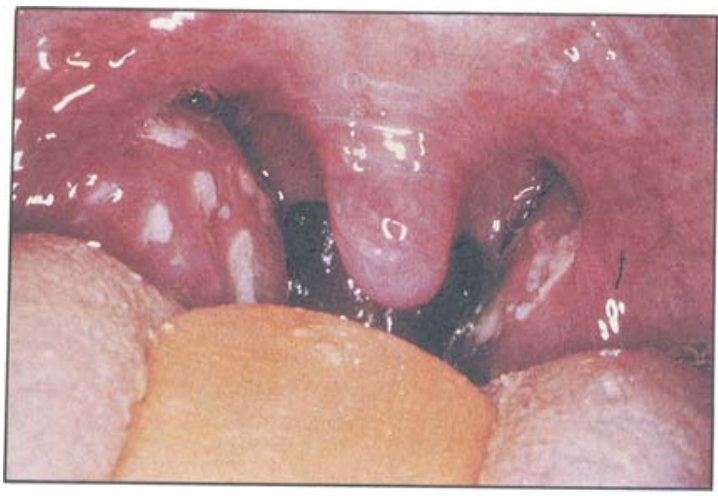

Fig. 1. Exudative pharyngotonsillitis.

The most common causes of sore throat are infections primarily involving the pharynx. Pain is aggravated by swallowing and may radiate along the distribution of the glossopharyngeal nerve to the ears. Associated features commonly include rhinorrhoea, cough, tender cervical lymphadenopathy, tonsillar enlargement, pharyngeal or tonsillar exudate (fig. 1) and fever. Although the symptoms and signs of pharyngitis are usually self-limiting within a few days they are among the most common reasons for seeking medical attention. This is a sensible precaution on the part of patients since a minority of sore throats are due to treatable lifethreatening diseases (diphtheria, acute epiglottitis, peritonsillar abscess), are a warning of potential but preventable rheumatic fever (streptococcal pharyngitis), or a symptom of infectious mononucleosis which may run a relatively protracted course. Some patients will have put themselves at risk of contracting gonococcal pharyngitis, in others Candida oropharyngo-oesophagitis may be a manifestation of AIDS and in a few, noninfectious causes of pain such as tumours, foreign bodies, cricoarytenoid arthritis and thyroiditis may be diagnosed. The challenge for the physician is to recognise the minority of sore throats which require treatment given that up to $90 \%$ of patients will have viral infections. This is not easy as pathognomonic clinical features of treatable infections are exceptional and laboratory diagnosis is time-consuming and costly because of the numbers involved.

\section{Aetiology, Clinical Diagnosis and Management of Sore Throat}

Some of the causes of sore throat, the associated syndromes and their approximate prevalence are shown in table I. Certain characteristic clinical features sometimes provide a fast track to diagnosis despite this rather daunting list.

\subsection{Mild Sore Throat with Rhinorrhoea}

Irritation or 'scratchiness' of the throat with mild oedema and erythema of the pharynx is present in about $50 \%$ of those with the common cold syndrome due to rhinoviruses, coronaviruses or parainfluenza viruses. A minority of these patients, who are generally afebrile and whose principal complaint is rhinorrhoea, will consult their doctors and if a throat swab is taken it serves only to confuse. Five to $60 \%$ of healthy people are pharyngeal carriers of Streptococcus pyogenes and carriage of this organism is not a reason for treatment. Moreover, it is often-stated medical dogma that antibiotics are not effective in preventing those bacterial infections which occasionally follow viral upper respiratory tract disease. This has been amply demonstrated in some situations (David \& Wedgewood 1965; Maisel et al. 1968), although an open mind must be kept regarding particular bacterial complications of certain viral infections which may not have been subject to clinical trials, e.g. staphylococcal pneumonia during influenza epidemics.

\subsection{Acute Laryngitis}

Hoarseness and aphonia are usually due to viral upper respiratory infection but can, in perhaps $10 \%$ of cases, be caused by bacterial infections including streptococcal pharyngolaryngitis, diphtheria and Haemophilus epiglottitis (Glezen et al. 1967; Gwaltney 1990). In the patient without other features of these diseases and who is not severely ill it is unnecessary to do more than ensure that follow-up laryngoscopy is performed if symptoms persist beyond about 10 days.

It is the child under 5 years of age, and much 
Table I. Causes, syndromes and prevalence of sore throat

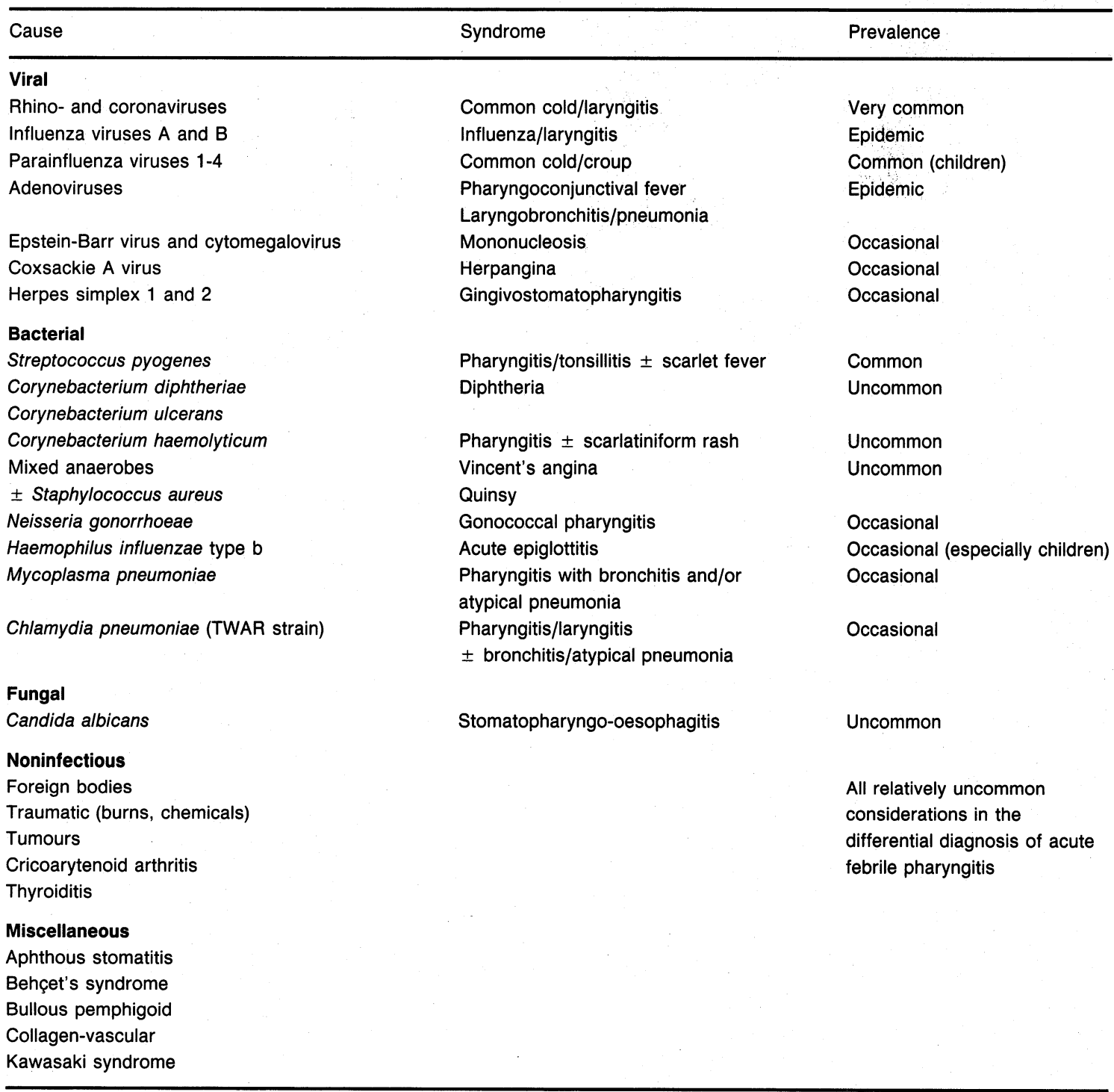

less commonly the older child or adult, who may present with acute epiglottitis due to Haemophilus influenzae type $\mathrm{b}$. Patients with this infection need particularly urgent and careful evaluation. There is associated high fever and dysphagia with characteristic drooling. About $20 \%$ have a cough. A cherryred, oedematous epiglottis, rather than pharyngitis, is the cardinal sign but the diagnosis is better made by an immediate lateral x-ray of the soft tissues of the neck and confirmed by obtaining blood cultures rather than a throat swab, since examination may precipitate complete airways obstruction. Acute epiglottitis is a medical emergency in which intubation is commonly required.

$H$. influenzae type $\mathrm{b}$ may produce $\beta$-lactamase, conferring resistance to ampicillin, and occasional 
strains are also resistant to chloramphenicol; thus, the antibiotic treatment of choice is cefuroxime or a third-generation cephalosporin (e.g. cefotaxime). The combinations of amoxicillin or ampicillin with a $\beta$-lactamase inhibitor (e.g. amoxicillin/clavulanic acid or ampicillin/sulbactam) would also be expected to be effective but are less well tried.

\subsection{Vesicles and Ulcers}

Primary infection with herpes simplex type 1 or 2 typically causes gingivostomatitis. Less often the characteristic painful superficial vesicles and ulcers set on an erythematous base extend beyond the palate and buccal mucosa to the pharynx, or are localised to the pharynx. This latter presentation is typical of young adults encountering herpes simplex type 1 virus for the first time, whereas children under the age of 5 years tend to be asymptomatic or present with gingivostomatitis. Fever and tender cervical adenopathy are commonly present for 1 or 2 weeks until spontaneous resolution occurs. In the immunocompromised patient reactivation may be associated with large, painful, chronic ulcers. If the clinical diagnosis is uncertain the virus can usually be grown in cell culture within 48 to 72 hours. Acyclovir is indicated in immunocompromised patients (Corey \& Spear 1986).

Herpangina (fig. 2) is a vesicular disease of the fauces and soft palate accompanied by fever, sore throat and pain on swallowing. It is most com-

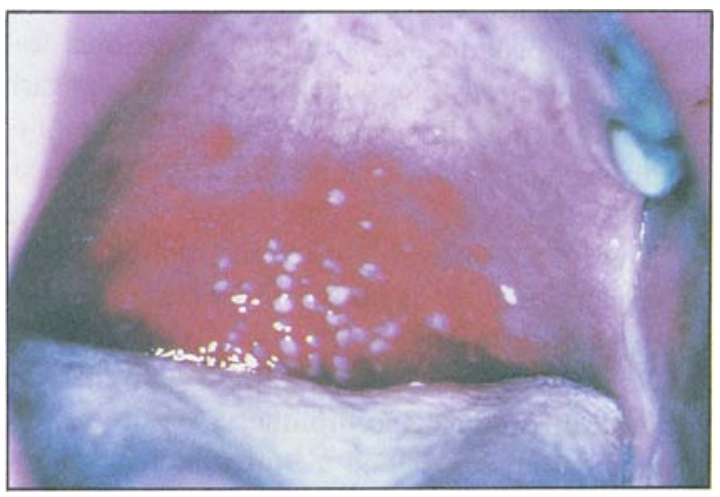

Fig. 2. Greyish-white vesicles surrounded by red areola in Coxsackie A herpangina.

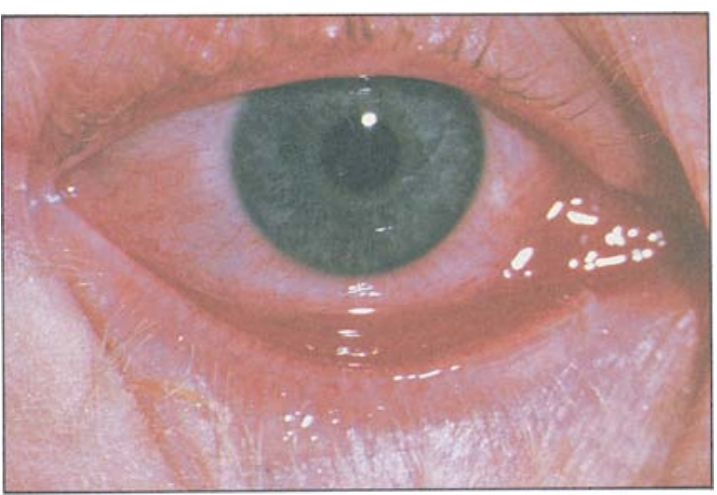

Fig. 3. Conjunctivitis associated with adenoviral pharyngitis.

monly caused by Coxsackie A viruses but may also be due to other enteroviruses including Coxsackie $B$ and certain echoviruses. Children of kindergarten and primary school age are primarily affected, sporadically or during epidemics in the summer months. Several members of a household may contract sore throats at intervals of a few days. Unlike primary herpes, gingivitis and cervical adenopathy do not occur. Confirmation of the diagnosis by isolating the virus from throat or faeces is sometimes possible, but is not routinely available. Prompt recovery occurs without specific therapy. Other types of ulceration, of uncertain cause, which may extend to the pharynx include aphthous ulcers, Behçet's disease and ulceration associated with severe neutropenia.

\subsection{Pharyngoconjunctival Fever}

Several adenoviruses can cause severe pharyngitis clinically indistinguishable from streptococcal pharyngitis except when there is an associated conjunctivitis, which occurs in 30 to $50 \%$ of sporadic cases. This follicular, usually unilateral conjunctivitis (fig. 3) seldom accompanies adenoviral pharyngitis occurring in closed communities such as military recruits.

\subsection{Pharyngitis with Influenza or Lower \\ Respiratory Tract Infection}

Pharyngitis is occasionally a prominent manifestation of influenza infection which is recognised readily enough during epidemics and may be sus- 


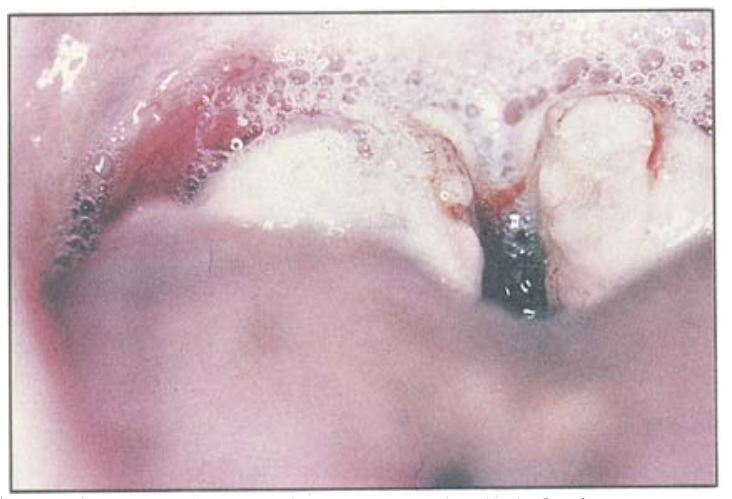

Fig. 4. Cheesy, creamy-white membrane in infectious mononucleosis.

pected at other times because of the severity of associated fever, myalgia and headache. Exudate and adenopathy do not occur. Lower respiratory complications such as influenzal pneumonia or secondary bacterial pneumonia are uncommon but may be life threatening. Recognition of influenza outbreaks is important because those at greatest risk can be protected by vaccination and/or amantadine.

Relatively mild pharyngitis is also a common manifestation of Mycoplasma pneumoniae infection and infection due to Chlamydia pneumoniae, previously called $C$. psittaci Strain TWAR (Grayston et al. 1986). Both these agents may also cause atypical pneumonia, but this is the tip of the iceberg as most infections are subclinical. Pharyngeal and tonsillar exudates and cervical lymphadenopathy may be seen with $M$. pneumoniae infection and rhinitis is common in infancy. Severe pharyngitis, often with laryngitis, is said to be suggestive of TWAR infection, and lower respiratory disease may not be apparent for a further week or more. Both illnesses can be confirmed serologically, or by culture where this is available. Both erythromycin and tetracycline are effective in the treatment of lower respiratory infection due to $M$. pneumoniae but their role in pharyngitis is not established (Shames et al. 1970). Tetracycline may be the antibiotic of choice in TWAR which does not appear to respond to erythromycin therapy (Schaffner 1990).
Although some adenoviruses are also common causes of pneumonia, they are usually different serotypes from those which cause pharyngitis.

\subsection{Membranous Pharyngitis}

The presence of a grey-green membrane, firmly adherent to the tonsils and pharyngeal mucosa, should always raise the suspicion of diphtheria even though this is uncommon in countries where an adequate vaccination programme is maintained. The principal differential diagnosis is infectious mononucleosis and clues outside the oropharynx generally allow these illnesses to be distinguished from one another. A mucopurulent nasal discharge and crusting of the nares may be seen with diphtheria. The pharyngeal membrane in diphtheria may cover the uvula and its extension to involve the larynx may produce hoarseness and stridor. Tender anterior cervical lymphadenopathy is usual and occasionally painful: inflammatory oedema produces a classic 'bull-neck' appearance. Evidence of myocarditis and peripheral neuritis should be sought. Haematological and biochemical findings are nonspecific.

In contrast, in infectious mononucleosis the cheesy, creamy-white exudate or membrane is confined to the tonsils (fig. 4), generalised lymphadenopathy (often with splenomegaly) is usual, liver function tests are disturbed, the peripheral blood shows a lymphocytosis with atypical mononuclear cells, and at some stage the heterophile antibody test (Monospot, Paul-Bunnell) is usually positive. Periorbital oedema and a rash are occasional features of infectious mononucleosis. A macular rash is seen in almost all patients with infectious mononucleosis inadvertently given ampicillin or amoxicillin (fig. 5).

The management of infectious mononucleosis is supportive; there is no specific therapy. Corynebacterium diphtheriae and Corynebacterium ulcerans, some strains of which are also toxigenic and which can produce the diphtheria syndrome, are readily cultured provided the microbiology laboratory is alerted to the possible diagnosis. However, when diphtheria is strongly suspected, antitoxin and 


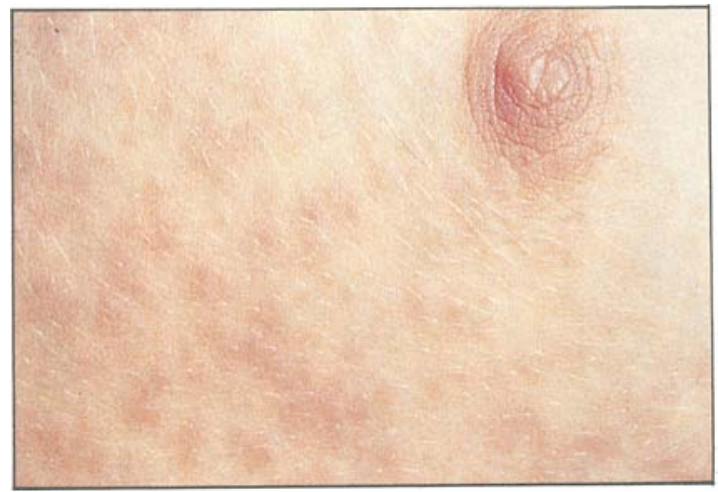

Fig. 5. Macular rash in a patient with infectious mononucleosis given ampicillin.

either penicillin or erythromycin should be given at once without waiting for bacteriological confirmation.

\subsection{Exudative Pharyngitis with a Rash}

The decline in prevalence of scarlet fever has greatly outstripped that of streptococcal pharyngitis, presumably because those strains of $S$. pyogenes infected with a lysogenic phage mediating erythrogenic toxic production are now relatively uncommon. The typical sunburn with goose pimples, circumoral pallor, white then red strawberry tongue, with convalescent desquamation, especially of the extremities (fig. 6), may never be seen by many practitioners. Moreover, as the frequency

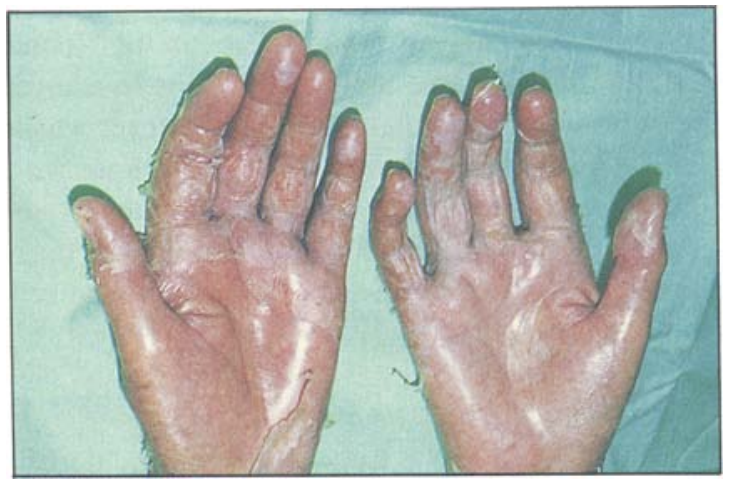

Fig. 6. Desquamation as seen after scarlet fever or staphylococcal toxic shock syndrome. of this disease has declined, the possibility that a patient with many of these features has one of the uncommon illnesses included in the differential diagnosis becomes more likely.

Corynebacterium haemolyticum has been described as a cause of pharyngitis and scarlatiniform rash in young adults, especially of teenage years, and in this age group a scarlet fever syndrome is almost as likely to result from $C$. haemolyticum as from $S$. pyogenes, or at least that is the experience reported from Seattle (Miller et al. 1986). Desquamation is less prominent following $C$. haemolyticum infection than following scarlet fever and the former is not associated with the sequelae of rheumatic fever or glomerulonephritis. A 10-day course of erythromycin is clinically and microbiologically effective treatment in most patients with C. haemolyticum pharyngitis, and is preferred to penicillin (Leading Article 1987).

Pharyngitis is one of the less dramatic of the many manifestations of staphylococcal toxic shock syndrome, a diagnosis which should be considered ahead of scarlet fever in the hypotensive patient, especially a menstruating woman who is using tampons. Antibiotic (flucloxacillin) therapy may have less to do with determining the immediate outcome than in preventing relapse a month later.

As has been mentioned, the Epstein-Barr virus causes a macular rash in 5 to $10 \%$ of patients with infectious mononucleosis and in over $90 \%$ of those given ampicillin or amoxicillin during the acute phase of the infection. This may occasionally lead to confusion with enteroviral infections which are also capable of causing pharyngitis and a rash.

\section{Streptococcal Pharyngitis}

When all the epidemiological and clinical clues are taken into account it is still most likely that the medical practitioner will be faced by a patient with nonspecific pharyngitis with or without an exudate or anterior cervical adenopathy. The question which must be answered is whether this is due to $S$. pyogenes. Streptococcal pharyngitis should be adequately treated to prevent the complication of rheumatic fever, to cure the acute sore throat, pre- 
vent suppurative complications and to make the patient noninfectious to others. Most other cases of pharyngitis are of viral aetiology and antibiotic treatment is contraindicated because it is ineffective, has potential side effects and is not cost effective. Unfortunately, even the most experienced practitioner has only about a $75 \%$ chance of correctly diagnosing streptococcal pharyngitis on the basis of clinical and epidemiological findings (Peter 1986). It remains controversial how avidly the diagnosis of streptococcal pharyngitis should be pursued and there are several possible strategies.

\subsection{Investigation and Treatment Strategies}

\subsubsection{Positive Throat Swab Culture}

Throat swab culture identifies about $90 \%$ of those with $S$. pyogenes in the pharynx, i.e. about $10 \%$ of adults and $35 \%$ of children who present with pharyngitis in the nonepidemic situation. However, only about $50 \%$ of those harbouring $S$. pyogenes are actually infected, the remainder being incidental or chronic carriers who are not at risk themselves nor likely to infect others. The delay in obtaining results may minimise the effect of treatment on symptoms (which is minimal and evident only when treatment is begun early), may decrease the chance of preventing spread of the infection to others and finally runs the risk that the patient will not return, thereby missing treatment altogether.

Treatment may be started and subsequently stopped if cultures are negative. Unfortunately both doctor and patient compliance has been shown to be deficient in this strategy (Peter 1986). Alternatively, treatment can be started following a telephoned report of the culture result. Intriguing support for this approach comes from a study which showed that penicillin treatment delayed for 48 to 56 hours was associated with a lower rate of recurrence and did not increase intrafamilial spread when compared with treatment begun immediately (Pichichero et al. 1987).

Rapid diagnostic tests for $S$. pyogenes based on the detection of a protein constituent of the organism on the throat swab are commercially available. Some have been shown to have a specificity of over

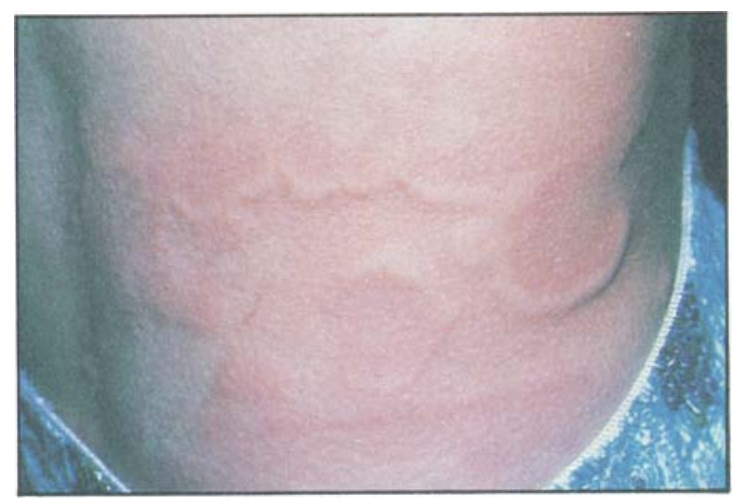

Fig. 7. Adverse reaction to a penicillin.

$95 \%$ and a sensitivity of about $90 \%$ for those swabs which would yield more than 10 colonies of $S$. pyogenes on culture (Halsted 1986). Since the clinical significance of lower numbers of colonies is uncertain, failure to detect antigen in these cases may not matter. Nevertheless, rapid methods remain less sensitive than a well-collected throat swab and it has been suggested that when they are negative, confirmation by culture is prudent (Kaplan 1988).

\subsubsection{Suspected S. pyogenes}

In this approach all sore throats are treated when there is a reasonable possibility they are due to $S$. pyogenes. This must remain an attractive option in areas where the incidence of both streptococcal pharyngitis and the complication of rheumatic fever are high and which at the same time lack readily accessible laboratory facilities. It should be remembered, however, that 1 to $2 \%$ of patients given penicillin will have an adverse reaction (fig. 7) and $0.001 \%$ will die (Idsoe et al. 1968). These risks must be balanced against that of rheumatic fever which follows in approximately 0.3 to $3 \%$ of those with untreated streptococcal pharyngitis. Blind prescribing is not easily defensible if an aetiological diagnosis could have been made with reasonable certainty.

\subsubsection{Selected Patients}

The third strategy is to treat, or culture and treat, selected patients (table II). An exudate, enlarged or tender cervical nodes and high fever $\left(\geqslant 38.3^{\circ} \mathrm{C}\right)$ all 
favour a streptococcal aetiology (Walsh et al. 1975). Pharyngitis is more likely to be of streptococcal aetiology in school-age children and this age coincides with the peak incidence of rheumatic fever (Peter 1986). Those presenting during a streptococcal epidemic, those who are contacts of patients with recently documented streptococcal infection and those with a past history of rheumatic fever are clearly candidates for treatment preferably in association with microbiological documentation.

A cost-effective analysis of various strategies conducted in the United States (Tomkins et al. 1977) found that in the epidemic situation it was medically most effective and least costly to treat all patients and that this was also true in the endemic situation where the positive throat culture yield was at least $20 \%$. Where the yield was below $5 \%$ it was more cost effective not to treat and for the intermediate situation treatment on the basis of a positive throat culture was most cost effective. The study also concluded, however, that for the individual patient the best strategy might depend on clinical findings. In the absence of detailed local information on epidemiology and the cost of laboratory tests and antibiotic therapy - all of which are constantly changing variables - it is difficult to lay down universally acceptable guidelines and the 'art' of medicine continues to have its place.

\subsection{Therapeutic Regimens}

Acceptable regimens are oral phenoxymethylpenicillin $250 \mathrm{mg} 4$ times daily for 10 days, oral erythromycin estolate $250 \mathrm{mg} 4$ times daily for 10

Table II. Management guidelines for possible streptococcal pharyngitis

Administer penicillin for 10 days (unless culture is negative) if:

- History of rheumatic fever

- School age

- Contact with S. pyogenes infection

- Hyperendemic population

- Temperature $\geqslant 38.3^{\circ} \mathrm{C}$

- Exudate

- Cervical lymphadenopathy

- Scarlatiniform rash

If none of the above, give symptomatic treatment unless culture (optional) is positive days or a single injection of benzathine penicillin 1.2 million units. Shorter courses of treatment with penicillin have been shown to have an increased bacteriological failure rate and cannot be recommended (Gerber et al. 1987). Phenoxymethylpenicillin or erythromycin estolate can also be given as $500 \mathrm{mg}$ twice daily, but this leaves less margin for error if a dose is forgotten. Cephalosporins or clindamycin are also effective. There is no reason to prescribe ampicillin or amoxicillin for streptococcal pharyngitis.

\section{Conclusion}

The cause of sore throat should be established when possible because treatment is important in selected cases: these include streptococcal pharyngitis (to prevent rheumatic fever), diphtheria, Haemophilus epiglottitis, peritonsillar abscess and gonococcal pharyngitis. The history and clinical findings in cases of sore throat are only occasionally pathognomonic but examination should include other systems as well as the throat. In most cases the important distinction is between streptococcal and viral pharyngitis and this cannot be made reliably on clinical grounds alone.

Strategies for managing suspected streptococcal pharyngitis range from empirical treatment of all cases to treatment only after confirmation by laboratory culture. The cost-effectiveness of various strategies depends on the incidence of streptococcal infection and rheumatic fever in the community and on the costs of diagnostic tests and antibiotic therapy. A correctly collected and processed throat culture remains more sensitive than the numerous rapid antigen tests developed over the past decade, but the latter are especially helpful if positive. Penicillin given for 10 days remains the treatment of choice for streptococcal pharyngitis.

\section{References}

Corey L, Spear PG. Infections with herpes simplex viruses. New England Journal of Medicine 314: 686-691, 749-757, 1986

Davis SD, Wedgewood RJ. Antibiotic prophylaxis in acute viral respiratory disease. American Journal of Diseases of Children 109: 544-553, 1965

Gerber MA, Randolph MF, Chanatry J, Wright LL, DeMeo K, 
et al. Five vs ten days of penicillin $\mathrm{V}$ therapy for streptococcal pharyngitis. American Journal of Diseases in Children 141: 224-227, 1987

Glezen WP, Clyde WA, Senior RJ, Sheaffer CI, Denny FW. Group A streptococci, mycoplasmas and viruses associated with acute pharyngitis. Journal of the American Medical Association 202: 119-124, 1967

Grayston JT, Kuo CC, Wang SP, Altman J. A new chlamydia psittaci strain, TWAR, isolated in acute respiratory tract infections. New England Journal of Medicine 315: 161-168, 1986

Gwaltney JM. Acute laryngitis. In Mandell et al. (Eds) Principles and practice of infectious diseases, 3rd ed. Churchill Livingston, New York, 1990

Halsted CC. Rapid tests for group A streptococcus: unsettled issued. Infectious Diseases Newsletter 5: 83-84, 1986

Idsoe $\mathrm{O}$, Guthe T, Wilcox RR, et al. Nature and extent of penicillin side reactions with particular reference to fatalities from anaphylactic shock. Bulletin of the World Health Organization 38: $159-188,1968$

Kaplan EL. The rapid identification of group A beta-hemolytic streptococci in the upper respiratory tract: current status. New Topics in Paediatric Infectious Diseases 35: 535-542, 1988

Leading Article. Bacterial pharyngitis. Lancet 1: 1241-1242, 1987

Maisel JC, Pierce WE, Stille WT. Chemoprophylaxis. American Reviews of Respiratory Disease 97: 366-375, 1968

Miller RA, Brancato F, Holmes KK. Corynebacterium haemo- lyticum as a cause of pharyngitis and scarlatiniform rash in young adults. Annals of Internal Medicine 105: 867-872, 1986

Peter G. The child with Group A streptococcal pharyngitis. In Arnoff SC (Ed.) Advances in paediatric infectious diseases, Year Book Medical Publishers Inc., Chicago, 1986

Pichichero ME, Disney FA, Talpeg WB, Green JL, Francis AB, et al. Adverse and beneficial effects of immediate treatment of group A beta-hemolytic streptococcal pharyngitis with penicillin. Pediatric Infectious Disease Journal 6: 635-643, 1987

Schaffner W. TWAR. In Mandell et al. (Eds) Principles and practice of infectious diseases, 3rd ed., Churchill Livingston, New York, 1990

Shames JM, George RB, Holliday WB, Rasch JR, Mogabgab WJ. Comparison of antibiotics in the treatment of mycoplasmal pneumonia. Archives of Internal Medicine 125: 680-684, 1970

Tomkins RK, Burnes DC, Cable WE. An analysis of the costeffectiveness of pharyngitis management and acute rheumatic fever prevention. Annals of Internal Medicine 86: 481-492, 1977

Walsh T, Bookheim WW, Johnson RC, Tomkins RK. Recognition of streptococcal pharyngitis in adults. Archives of Internal Medicine 135: 1493-1497, 1975

Correspondence and reprints: Dr S.D.R. Lang, Middlemore Hospital, Otahuhu, Auckland 6, New Zealand.

\section{Errata}

Vol. 39, No. 6, 1990: To better reflect the current database for oropharyngeal and oesophageal candidiasis, the following corrections to the article on fluconazole should be noted:

Page 880, 'Dosage and Administration' section, first paragraph, second sentence, replace with 'The usual recommended dosage in patients with oropharyngeal and oesophageal candidiasis is 50 to $100 \mathrm{mg}$ once daily for 1 to 4 weeks.'

Page 896, third paragraph, first sentence, replace with 'Most studies employed a dosage of $50 \mathrm{mg}$ orally once daily for 1 to 4 weeks although $100 \mathrm{mg}$ orally once daily has been used in an attempt to prolong the disease-free interval.'

Page 910, sixth paragraph, replace this paragraph with 'The usual recommended dosage in patients with oropharyngeal and oesophageal candidiasis is 50 to $100 \mathrm{mg}$ once daily for 1 to 4 weeks.'

Page 911, 'Place of Fluconazole in Therapy', first paragraph, third sentence, replace with 'Although initial studies related success with low dosages $(50 \mathrm{mg} /$ day) of the drug, $100 \mathrm{mg} /$ day has also been successful in the treatment of patients with oropharyngeal candidiasis. Patients with AIDS may require maintenance therapy.' 